\title{
A QUALIDADE DE SERVIÇOS MÉDICOS NA PERSPECTIVA DO CLIENTE
}

\author{
André Torres Urdan \\ Mestre em Administração pela FGV-EAESP, Doutor em Administração pela FEA-USP, \\ Professor Adjunto do Departamento de Mercadologia da FGV-EAESP e Pesquisador do CNPq. \\ E-mail: aturdan@fgusp.br
}

\begin{abstract}
A qualidade de serviços de cuidado à saúde compreende dois domínios: técnico e interpessoal. Os pacientes quase sempre conhecem pouco sobre os aspectos da qualidade técnica, mas, em geral, não têm dificuldade em avaliar o lado interpessoal. Falta, no Brasil, porém, instrumental para a mensuração da Qualidade Experimentada pelo Paciente (QEP), razão pela qual o objetivo aqui foi analisar empiricamente propriedades psicométricas de uma escala desse construto no tocante aos serviços médicos de consultório. Assomaram-se seis fatores subjacentes à QEP, englobando 40 atributos, cobrindo os domínios técnico e interpessoal. Quanto à fidedignidade, ela é apropriada em todas as seis escalas dos fatores prevalecentes neste estudo para a QEP. No que concerne à validade, ela é destacada para as escalas de cinco desses seis fatores. Seguem, ainda, as limitações e implicações da pesquisa.
\end{abstract}

RESUMO

\section{ABSTRACT}

Health care service quality involves two domains: technical and interpersonal. Patients almost always know very little about the aspects of technical quality, but in general they don't face difficult evaluating the interpersonal quality. However, in Brazil, it is missing a tool to measure the Quality Experienced by the Patient (QEP). Thus, the goal of this research was to undertake an empirical analysis of the psychometric properties of a scale of QEP regarding the office medical services. The results revealed six factors underlying QEP, comprising the domains of technical and interpersonal quality. About reliability, it seemed adequate in all the scales of the six factors. Finally, concerning validity, it is impressive for five out of six factors. The limitations and implications

of the research are also presented.

\section{PALAVRAS-CHAVE}

Qualidade percebida, serviços, pacientes, médicos, consultório.

\section{KEY WORDS}

Perceived quality, services, patients, physicians, office. 


\section{INTRODUÇÃO}

No Brasil, os médicos enfrentam a difícil realidade de deterioração do sistema público de saúde, elevada dependência de organizações privadas do setor - como as de medicina de grupo e seguros -, pacientes com níveis crescentes de exigência e incremento da concorrência entre profissionais. Nesse panorama, destaca-se a qualidade de serviço, uma vez que o paciente, apesar de certas dificuldades, não deixa de avaliá-la quando se trata de cuidados à saúde. Mas ainda falta melhor compreensão sobre a percepção do paciente a respeito dessa esfera de qualidade, mormente um conhecimento que reflita a realidade brasileira, na qual pode haver especificidades diferentes de outros países. Eis o problema contemplado neste artigo, com foco no trabalho do médico no seu consultório com os pacientes, mediante uma abordagem teórico-empírica.

\section{BREVE REVISÃO TEÓRICA}

Doravante, discorrer-se-á sobre a essência da literatura sobre os tópicos: a) qualidade de serviço e qualidade percebida; b) qualidade de serviços de cuidado à saúde e o paciente e c) qualidade percebida pelo paciente em serviços médicos de consultório. Desse modo, partindo do geral para o específico, ter-se-á base teórica para a análise empírica do tema.

\section{Qualidade de serviço, qualidade}

percebida e o Modelo de Grönroos

A pesquisa sobre qualidade de serviço salienta a Qualidade Percebida (QP), baseada no cliente. Tanto para serviços quanto para bens, a QP envolve uma avaliação, subjetivamente realizada pelo cliente, de excelência ou superioridade de uma oferta (Garvin, 1984). A percepção é um processo de organização, interpretação e derivação de significado de estímulos por meio dos sensos (Monroe e Krishnan, 1985). Sensação é um processo de recebimento dessas impressões sensoriais. Logo, a percepção é subjetiva. A cognição do cliente sobre um estímulo (seja serviço ou bem) não é uma foto e sim um desenho, que exprime sua visão individual da realidade. Isso torna imprescindível discernir entre atributos de uma oferta por si e as percepções dos clientes sobre eles, porque os clientes podem diferir, e muito, em suas percepções e estas afetam o comportamento, não os atributos em si (Howard, 1977).

Um interessante modelo genérico da QP de serviço é o de Grönroos (1990), apresentado na Figura 1. Nele a QP resulta da comparação entre a qualidade esperada e a qualidade experimentada pelo cliente. A QP é boa quando a qualidade experimentada ultrapassa (ou ao menos alcança) as expectativas do cliente (qualidade esperada). No modelo, são duas as dimensões de qualidade: técnica e funcional. A qualidade técnica é o que o cliente absorve ao terminar sua interação com o prestador de serviços e os processos de produção e entrega. Sendo os serviços processos experienciais (pois atividades de produção e consumo ocorrem simultaneamente), o modo como transcorrem as interações tem impacto sobre a percepção do serviço. Disso surge a qualidade funcional: a maneira como a qualidade técnica - o resultado final do processo - é transferida ao cliente. A avaliação funcional inclina-se a ser mais subjetiva que a dimensão técnica. Ademais, há a imagem do prestador de ser-

Figura 1 - Modelo Genérico de Qualidade de Serviço de Grönroos

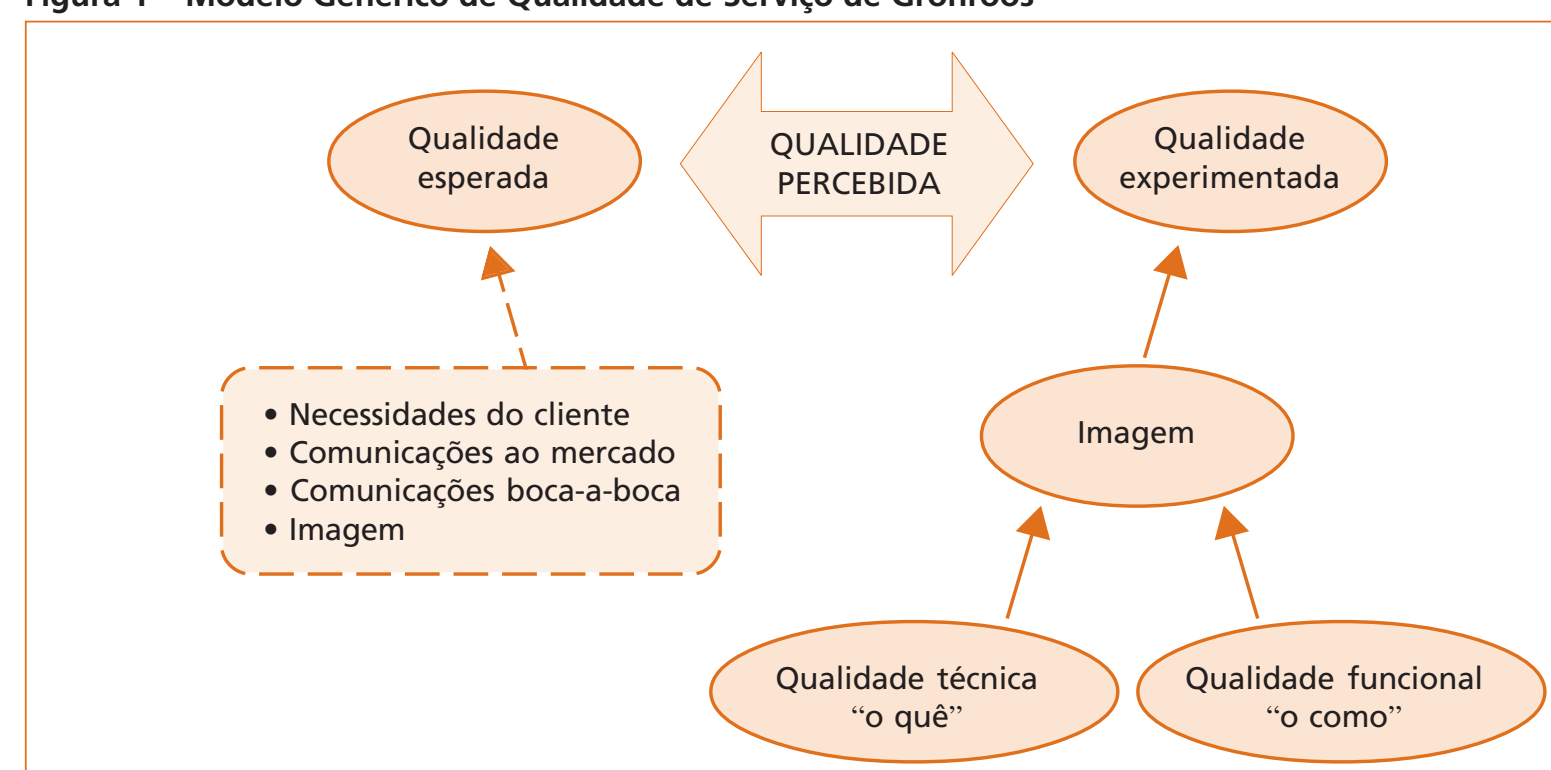

Fonte: Grönroos (1990, p. 41). 
viços, consistindo em valores a ele conectados pelos clientes - atuais, potenciais, perdidos -, que opera como um filtro dos atributos que influenciarão a avaliação da qualidade experimentada.

\section{Qualidade de serviços de}

cuidado à saúde e o paciente

É preciso mapear eventuais particularidades da qualidade de serviços de cuidado à saúde (Ford, Bach e Fottler, 1997). Pairam certas dúvidas sobre a transferibilidade, isto é, se os princípios e técnicas genéricos de qualidade de serviços podem ser aproveitados nas atividades de cuidado à saúde. As organizações de saúde têm muito em comum com as de vários outros ramos de serviços, mas três singularidades as marcam (Garvin, 1990): a) falta de clareza na conexão entre entradas e saídas, muito em função do tempo necessário para os resultados manifestarem-se; b) os pacientes podem ter dificuldade em avaliar aspectos técnicos; c) os grandes hospitais operam com

\section{O PACIENTE AVALIA A QUALIDADE}

\section{TÉCNICA DO CUIDADO}

\section{INDIRETAMENTE PELAS EVIDÊNCIAS \\ DO INTERESSE E DA PREOCUPAÇÃO}

\section{DOS PROFISSIONAIS COM SUA}

\section{SAÚDE E SEU BEM-ESTAR.}

duas linhas de autoridade distintas, o pessoal administrativo e os médicos, em vez da pirâmide de autoridade única predominante nas demais organizações. Além disso, como em tantos outros ramos, serviços de saúde têm vários tipos de clientes (Berwick, Godfrey e Roessner, 1990). Um pediatra, por exemplo, pode prestar serviço à criança, à família dela, à comunidade, a outros médicos, a outros profissionais de saúde, a quem paga pelos serviços e até ao seu conselho e às suas entidades de classe.

A qualidade de serviços de cuidado à saúde abarca aquilo que possa aumentar uma medida abrangente de bem-estar do paciente, após considerar-se o balanço de ganhos e perdas esperados, intervenientes em todas as partes do processo (Donabedian, 1980). Na concepção consagrada desse autor, tal qualidade compreende dois domínios: técnico e interpessoal. O técnico abrange a aplicação da ciência e da tecnologia da Medicina à administração de um problema pessoal de saúde. Já o interpessoal refere-se à administração da interação social e psicológica entre o cliente e os profissionais. Ao domínio interpessoal são somadas ainda as amenidades, formadas pelas propriedades do ambiente onde o cuidado é propor- cionado - como uma sala de espera agradável -, gerando atributos como conforto, presteza e cortesia.

Os pacientes quase sempre conhecem bem pouco os detalhes do domínio técnico da qualidade, como ressalta Donabedian (1985), embora apreciem sua importância, especialmente em situações que tragam clara ameaça à saúde e ao bem-estar. Em geral, o paciente avalia a qualidade técnica do cuidado indiretamente, pelas evidências do interesse e da preocupação dos profissionais com sua saúde e seu bem-estar. Mas esse autor evidencia como o paciente usualmente não tem dificuldade em avaliar a qualidade interpessoal, configurada nas condições sob as quais o cuidado é prestado e a maneira como é tratado pelos profissionais. De mais a mais, o gabarito do domínio interpessoal pode influenciar os resultados do domínio técnico para o paciente e vice-versa. Um domínio não é eficaz sem o outro (Donabedian, 1985). O relacionamento interpessoal e o conforto das amenidades não curam doenças orgânicas. A competência técnica - a menos que ela seja em prol do paciente em virtude do interesse humano e do comprometimento dos profissionais em prover os melhores benefícios ao paciente - também não é por si só suficiente.

Esse esquema parece demonstrar que os serviços de saúde não fogem, em essência, do caráter genérico da qualidade de serviço. A distinção proposta por Grönroos (1990) entre as qualidades técnica e funcional mostra-se válida tratando-se de serviços de saúde. A qualidade técnica refere-se ao domínio técnico dos serviços de cuidado à saúde, ao passo que a qualidade funcional reflete seu domínio interpessoal.

A qualidade funcional toma, no entanto, contornos desafiadores. Clark (2001) menciona que muitas escolas de medicina nos Estados Unidos estão mudando seus currículos para realçar as qualidades humanísticas inerentes ao trabalho médico. Tais mudanças são, contudo, apenas o primeiro passo para transformar um modelo de formação desnecessariamente cruel, que amortece as emoções e desumaniza o médico. Um caminho melhor é sugerido, em que os estudantes aprendam, por exemplo, anatomia e habilidades de comunicação com o paciente ao mesmo tempo, de modo a saber que o fígado que analisam pertence sempre a um ser humano.

Em outro prisma, resultados interessantes surgem de ampla pesquisa sobre qualidade de cuidados de saúde realizada com médicos da Austrália, do Canadá, dos Estados Unidos, da Grã-Bretanha e da Nova Zelândia (Blendon et al., 2001). Para o aprimoramento dessa qualidade, a maioria dos médicos pesquisados foi a favor de reformas para que tenham mais tempo com seus pacientes, mas só de 33\% a 40\% dos inquiridos concordavam que avaliações do paciente poderiam servir ao mesmo propósito. Isso mostra pouca disposição desses médicos em acolher o feedback do paciente, apesar do interesse em poder-lhes dedicar mais tempo. 
Posto isso, torna-se não só admissível, mas recomendável, indagar aos pacientes como melhorar a qualidade dos serviços de saúde. Resta suprir a falta de instrumental para a mensuração da QP pelo paciente, sobretudo diante da considerável agregação de serviços prestados pelos profissionais e organizações de saúde. Sem isso, torna-se muito difícil a orientação das equipes de saúde em relação ao leque de atributos que conformam a qualidade sob a ótica do paciente. Repare-se que somente agora o Comitê Nacional para a Asseguração de Qualidade dos Estados Unidos decidiu desenvolver medidas de qualidade do trabalho médico baseadas também na perspectiva do paciente, exigindo o projeto quase dois anos de pesquisas (Pretzer, 2001). Logo, impõe-se avançar no desenvolvimento desse instrumental também no Brasil.

\section{Qualidade de serviços médicos de consultório percebida pelo paciente}

Dentro do amplo espectro de serviços de cuidado à saúde, foca-se, aqui, a qualidade de serviços médicos de consultório percebida pelo paciente (QPC). Isso porque a maior parte do trabalho da classe médica tende a acontecer em consultórios, e a interação médico-paciente costuma ser muito duradoura em torno do espaço profissional do consultório. O trabalho teórico-empírico de Brown e Swartz (1989) - mesmo tantos anos depois - é proeminente no desenvolvimento de uma escala da QPC. Sobretudo porque eles, em vez de aproveitar o conteúdo de 22 itens da escala genérica de serviços Servqual (como O'Connor, Trinh e Shewchuk, 2000), identificaram variáveis específicas da QPC, cuja precisão decorrente aumenta a capacidade de compreensão e a ação profissional. A operacionalização da QPC desses dois autores abrangeu 65 atributos, para os quais uma análise fatorial revelou nove fatores subjacentes: Profissionalismo, Comunicações Auxiliares, Responsabilidade Profissional, Interações do Médico, Interações de Auxiliares, Diagnóstico, Competência Profissional, Comodidade de Horários e Comodidade de Localização.

Brown e Swartz (1989), todavia, distinguiram os domínios de expectativas e experiências de pacientes, cada qual retratado com um grupo, em parte próprio, de atributos. Eles registram, no artigo, que a comparação entre expectativas e experiências "só pôde ser computada para seis atributos" (p. 94), mas nada explicam sobre as razões de utilizarem apenas 6 dos 43 atributos distintos nesse cálculo. É estranho como tais autores definiram três conjuntos de atributos: um relacionado a expectativas, outro a experiências e um terceiro de atributos comuns a expectativas e experiências. Pode-se inferir que, para eles, há só uns poucos atributos, esses seis, pertencentes simultaneamente aos domínios particulares de expectativas e experiências dos pacientes. Aqui começam as críticas a tal trabalho científico.
A primeira delas reside em que, na concepção de autores como Parasuraman, Zeithaml e Berry (1985) e Grönroos (1990), a QP é expressa pela diferença entre expectativas e experiências dos clientes, logicamente com ambas as avaliações ocorrendo sobre os mesmos atributos. Por isso, afigura-se improcedente a separação promovida por Brown e Swartz (1989).

A segunda crítica corresponde à própria operacionalização da QP como a diferença entre experiências (desempenho) e expectativas. Há várias evidências de que o cálculo dessa diferença de escores é problemático ou melhores informações podem ser obtidas sobre a QP pelo exame somente das expectativas ou sobretudo das experiências. Demonstra-se isso em pesquisas empíricas genéricas sobre serviços no exterior (Cronin e Taylor, 1992) e no Brasil (Urdan e Magro, 1996; Johnston e Luce, 1996) como também em investigações específicas sobre serviços de saúde (Schewchuk, O'Connor e White, 1991; Taylor e Cronin, 1994). Em acréscimo, esse último esquema de cálculo acarreta a redução à metade da quantidade de avaliações que o cliente tem de realizar, ajudando na obtenção de colaboração por parte dele. Por conseguinte, entende-se que o melhor é a mensuração da QP baseada apenas em experiências.

\section{A Qualidade Percebida \\ É BOA QUANDO A QUALIDADE}

\section{EXPERIMENTADA ULTRAPASSA}

\section{AS EXPECTATIVAS DO CLIENTE.}

A terceira crítica consiste em que Brown e Swartz (1989) preocuparam-se em contar com atributos espelhando os dez fatores genéricos sugeridos originalmente por Parasuraman, Zeithaml e Berry (1985), com fundamento em estudos apenas qualitativos. Porém estes últimos, em uma segunda etapa quantitativa, passaram a advogar a existência de cinco fatores sob tal qualidade (Parasuraman, Zeithaml e Berry, 1988), embora sem tratarem de clientes de serviços de saúde. Portanto, Brown e Swartz (1989) tomaram por base uma dimensionalidade genérica da QP de serviços improcedente.

A quarta crítica a eles é dirigida ao fato de examinarem a validade convergente dos fatores da QPC tendo por base uma variável "satisfação global" do paciente como dependente em uma análise de regressão múltipla. Entretanto, a literatura é predominante em diferenciar os construtos QP e satisfação do consumidor. Mesmo tendo-se essa satisfação também operacionalizada como a diferença entre expectativas e experiências, eles não deixam de ter significados diferentes (Bolton e Drew, 1991a; La'Barbera e Mazursky, 1983; Oliver, 1997). Para este último (p. 162- 
188), a satisfação é uma resposta imediata ao consumo, enquanto a QP existe antes e após o consumo como um sinal duradouro da excelência de um bem ou serviço. Sobre a relação entre os dois construtos, há controvérsias, uma vez que o próprio Oliver (1997) considera que a qualidade antecede satisfação, ao passo que outros, como Bolton e Drew (1991b), sustentam uma ordem inversa. De qualquer modo, Brown e Swartz (1989) deveriam ter tomado como variável dependente em suas análises também uma medida global de qualidade (Taylor, 1994).

Apesar dessas críticas, o melhor da contribuição de Brown e Swartz (1989) parece estar no conjunto de atributos representando a QPC. Entende-se valer a pena aproveitar esse referencial como ponto de partida, submetendo-o às devidas análises. Sem levar em conta os fatores a que estavam conectados, os 43 atributos desses autores parecem, na face, exprimir o domínio da QPC. Neste artigo, contudo, considera-se que o total de atributos de expectativas e experiências no esquema de Brown e Swartz (1989), na realidade, exprime o todo da $\mathrm{QP}$, mesmo sendo esta aqui operacionalizada apenas com base em experiência.

\section{UMA INVESTIGAÇÃO EMPÍRICA}

A partir da teoria examinada, o objetivo empírico é analisar propriedades psicométricas de uma escala de QPC, formada pela consolidação dos atributos propostos por Brown e Swartz (1989), em uma operacionalização baseada apenas em experiência. De modo mais específico, o objetivo desdobrou-se em três questões a pesquisar, enunciadas no Quadro 1. Para respondê-las, implementouse uma pesquisa conclusiva e descritiva, de seção cruza- da (Churchill, 1991). Não foi possível, porém, gerar amostras representativas de amplas populações - fruto da restrição de recursos financeiros. Todas as análises foram implantadas em pacientes de consultório. As técnicas estatísticas aplicadas no exame de cada uma das questões constam do Quadro 1.

Um questionário foi elaborado com questões de avaliação dos 43 atributos de QPC, em uma escala de diferencial semântico com cinco posições (variando de " 1 qualidade muito baixa" a " 5 - qualidade muito alta"). Para o teste da terceira questão, foram acrescentadas: a) uma variável de Avaliação Global da QPC, com escala de diferencial semântico de cinco posições (variando de "1 qualidade global baixíssima" a "5 - qualidade global altíssima") e b) uma variável de Satisfação Global com Serviços de Consultório, com escala de diferencial semântico de cinco posições (variando de " 1 - absoluta insatisfação global" a "5 - absoluta satisfação global"). Tomou-se a escala de diferencial semântico como intervalar (Tull e Hawkins, 1990; Kinnear e Taylor, 1991), ensejando o uso de técnicas paramétricas. Uma bateria final do questionário continha algumas questões sobre o perfil sociodemográfico do respondente e sua relação com o plano de saúde. O formato final do questionário foi alcançado após duas séries de pré-teste, cada qual abrangendo oito pacientes, em especial porque não fora possível realizar uma tradução reversa do enunciado dos 43 atributos de QCP do português para o inglês.

Era preciso chegar aos pacientes a partir dos médicos com quem se consultavam. Isso foi tentado sem sucesso com o Conselho Regional de Medicina de Minas Gerais, a Cooperativa Unimed de Belo Horizonte, o Plano Santacasa Saúde da Santa Casa de Misericórdia de Belo

\section{Quadro 1 - Questões formuladas para a pesquisa e respectivas técnicas estatísticas}

\begin{tabular}{|c|c|}
\hline Questão & Técnica estatística \\
\hline $\begin{array}{l}1^{\text {a }} \text { - Há uma estrutura de QPC formada pelos } 9 \\
\text { fatores, ao longo dos } 43 \text { atributos, consolidados da } \\
\text { proposição de Brown e Swartz (1989)? Caso contrário, } \\
\text { quantos e quais são os fatores prevalecentes neste } \\
\text { estudo para a QPC? }\end{array}$ & $\begin{array}{l}\text { Análise fatorial, que enseja conhecer a estrutura de } \\
\text { correlações existentes entre grande número de va- } \\
\text { riáveis e representar os relacionamentos entre elas } \\
\text { em um conjunto reduzido de fatores subjacentes } \\
\text { (Hair Jr. et al., 1998). }\end{array}$ \\
\hline $\begin{array}{l}\mathbf{2}^{\mathbf{a}} \text { - São fidedignas as escalas da QPC, formadas } \\
\text { pelas variáveis dos atributos propostos por Brown } \\
\text { e Swartz (1989), para os fatores prevalecentes neste } \\
\text { estudo? }\end{array}$ & $\begin{array}{l}\text { Coeficiente alfa de Cronbach de consistência inter- } \\
\text { na, revelando o quanto os itens de uma escala es- } \\
\text { tão inter-relacionados. Se um conjunto de itens } \\
\text { representa uma mesma dimensão, eles têm de ser } \\
\text { consistentes na indicação que fazem dessa dimen- } \\
\text { são (Nunnaly, 1978). }\end{array}$ \\
\hline $\begin{array}{l}3^{a} \text { - Há validade nas escalas dos fatores da QPC } \\
\text { prevalecentes neste estudo, formadas pelas variá- } \\
\text { veis dos atributos propostos por Brown e Swartz } \\
(1989) ?\end{array}$ & $\begin{array}{l}\text { Análise de regressão linear múltipla, contemplan- } \\
\text { do o relacionamento entre uma variável dependen- } \\
\text { te e duas ou mais variáveis independentes, consis- } \\
\text { tindo em uma combinação linear das variáveis in- } \\
\text { dependentes que melhor predizem a variável de- } \\
\text { pendente (Perrien, Chéron e Zins, 1986). }\end{array}$ \\
\hline
\end{tabular}


Horizonte e a gestora Forluz do plano de medicina de grupo da Companhia Energética de Minas Gerais (Cemig). Ficou óbvio que a avaliação de serviços médicos por pacientes ainda é vista como algo bastante melindroso por essas organizações. Por fim, a Caixa de Assistência à Saúde (Casu) aceitou apoiar a pesquisa. A Casu é um plano de medicina de grupo do pessoal da Universidade Federal de Minas Gerais, do Centro Federal de Ensino Tecnológico de Minas Gerais, da Faculdade Federal de Odontologia de Diamantina e de suas respectivas fundações, associações e sindicatos.

No cadastro da Casu, foram obtidos 35 médicos, que tinham realizado pelo menos 30 consultas em pacientes seus nos dois meses anteriores e concordavam em participar da pesquisa. Seguiu-se a compilação de 35 listas, com nome e endereço de pacientes, para cada um dos 35 médicos participantes, totalizando 1.050 pacientes - selecionando-se os 30 últimos consultados por cada um dos médicos. Finalmente, ocorreu o levantamento de dados por via postal de setembro a novembro de 1998, com a remessa de uma carta de cobrança 20 dias após o envio do questionário.

\section{RESULTADOS}

Remetido o questionário aos 1.050 pacientes, 437 exemplares retornaram. Destes, 93 questionários foram descartados por terem mais de $20 \%$ das questões em branco ou todas as respostas em um extremo ou outro das escalas, denotando irreflexão. Restaram 344 questionários aproveitáveis, em uma proporção efetiva de retorno de $32,8 \%$ - interpretado como dentro do usual para levantamento postal. As análises foram realizadas no programa estatístico SPSS for Windows (versão 7.1).

Sobre o perfil geral da amostra, $86 \%$ dos pacientes realizaram duas ou mais consultas com o médico que avaliaram, ao passo que $38 \%$ realizaram seis ou mais consultas. Do total, 95\% dos pacientes tinham seis meses ou mais de vinculação à Casu e nenhum tinha um mês ou menos do total tempo de vinculação. Ademais, 54\% dos pacientes já haviam realizado dez ou mais consultas com médicos da Casu em geral, enquanto outros $29 \%$ tiveram de seis a dez consultas e nenhum fez apenas uma consulta. Isso tudo sugere uma amostra de pacientes com razoável vivência com o médico cujos serviços avaliaram e com a Casu, dando boa base para a avaliação de QPC. Do total, $72 \%$ eram mulheres, o que pode denotar maior boa vontade da parte delas do que dos homens em colaborar com a pesquisa. Verificou-se que $37 \%$ dos pacientes estavam entre 41 e 60 anos, enquanto outros 17\% tinham 61 anos ou mais, compondo um contingente de pessoas que devem possuir, pelo tempo de vida, uma experiência ao menos razoável como clientes - e não só como pacientes -, ajudando no desenvolvimento da capacidade de avaliação (inclusive por comparação com outras situações) de
QPC. Sobre nível de escolaridade, não mais que 23\% dos pacientes tinham pós-graduação. Tal perfil de escolaridade pareceu conveniente, pois deu maior variedade à amostra - ao contrário do receio que havia de aparecerem quase só docentes universitários.

\section{A MAIOR PARTE DO TRABALHO DA}

\section{CLASSE MÉDICA TENDE A ACONTECER}

\section{EM CONSULTÓRIOS, E A INTERAÇÃO}

\section{MÉDICO-PACIENTE COSTUMA}

\section{SER MUITO DURADOURA.}

As avaliações dos 43 atributos da QPC situaram-se em níveis majoritariamente elevados, com médias maiores que 4. Apenas 2 atributos (5\% do total) ficaram com média na faixa de 2 , e 8 atributos (19\% do total) ficaram com média na faixa de 3 . O panorama indicou uma elevada QPC nos médicos da Casu (4,1 de média das médias dos 43 atributos).

\section{Resultados da primeira questão}

Com relação à primeira questão, embora a maior parte das 43 variáveis dos atributos da QPC apresentasse assimetria, isso não comprometeu a realização da análise fatorial prevista. Nesta técnica, o efeito básico de fugas da normalidade, da homocedasticidade e da linearidade é basicamente a diminuição das correlações observadas (Hair Jr. et al., 1998, p. 99). O importante é que haja suficiente correlação entre as variáveis. Mas o valor da estatística de Bartlett (4947) e a medida do teste Kaiser-MeyerOkin $(0,91)$ sinalizam adequação dos dados para aplicar a técnica (Hair Jr. et al., 1998; Norusis, 1993).

Em um primeiro processamento por fatores comuns, definiu-se a priori a extração de nove fatores, com base na concepção teórica em teste. Nessa rodada, a média final das comunalidades (quantidade de variância que uma variável compartilha com todas as demais variáveis incluídas na análise) surgiu com considerável peso, no patamar de 0,64. Além desta, apenas a variável V34 ("Ser fácil e rápido marcar uma consulta com o médico") apresentou discrepância acentuada em face do panorama geral, com uma comunalidade final da ordem de 0,39 . Porém, os fatores 7,8 e 9 possuíam autovalores (variância total explicada por um fator) inferiores a 1 . Com a padronização, cada variável tem variância igual a 1 (Malhotra, 1996). Logo, não faz sentido reter um fator com autovalor inferior a 1, pois ele não é melhor do que uma variável isoladamente para explicar a variância presente. Por conseguinte, não há evidências para aceitar a existência de nove fatores subjacentes à QPC na amostra. 
Dessa forma, várias alternativas foram exploradas sobre o conjunto dos 43 atributos, tentando-se a extração pela fixação da quantidade de fatores tanto quanto o requisito de autovalores acima de 1, ao lado da investigação da plotagem de scree (Hair Jr. et al., 1998). A melhor solução foi com seis fatores da QPC, conforme mostrado na Tabela 1 , em que todos os fatores têm autovalor superior a 1, e há o mínimo de $60 \%$ de variância total explicada pelo conjunto de fatores em relação à variância existente no conjunto de variáveis originais. À primeira vista, o acentuado

\section{As AVALIAÇÕES HUMANAS}

\section{DE QUALIDADE SÃO SUBJETIVAS,}

\section{INFLUENCIADAS PELO}

\section{PARTICULAR REFERENCIAL DO}

\section{AVALIADOR COMO VALORES, CONHECIMENTOS E INTERESSES.}

degrau entre a variância explicada pelo primeiro fator $(42 \%)$ e o segundo (6\%) sugeria a unidimensionalidade da escala. Entretanto, após uma rotação ortogonal Varimax, a distribuição bem mais equilibrada das variâncias explicadas pelos fatores (22\% do primeiro e $12 \%$ do segundo) sinalizou a procedência da solução com os seis fatores, o que também ajuda a entender a estrutura dos dados com mais detalhes comparativamente à unidimensionalidade.

Ainda que não tenha prevalecido a solução com nove fatores, por trás desses seis estão agrupamentos de variáveis em fatores idênticos, ou quase isso, em oposição àqueles propostos originalmente por Brown e Swartz (1989). Este é o caso, agora, dos fatores 2, 3 e 6. Em acréscimo, os demais fatores $(1,4$ e 5) estão configurados simplesmente como a combinação de fatores da proposição desses dois autores. Para facilitar a interpretação, a Tabela 2 contém a matriz fatorial rotacionada incompleta (só coeficientes iguais ou maiores que 0,4 ) desses seis fatores, oferecendo um arranjo bem nítido e lógico. Somente os atributos V23, V35 e V43 não ficaram ligados a qualquer fator, pois têm cargas inferiores a 0,4 .

Por conseguinte, a resposta à primeira questão desta pesquisa é negativa, pois não parece haver uma estrutura formada por nove fatores subjacente à QPC. Ao contrário, na amostra, há evidências de que a QPC se desdobra em seis fatores: $1^{\circ}$ ) Interações do Médico e Diagnóstico; $2^{\circ}$ ) Competência Profissional; $3^{\circ}$ ) Interações de Auxiliares; $4^{\circ}$ ) Comodidade de Horários e Localização; $5^{\circ}$ ) Profissionalismo e Responsabilidade Profissional e $6^{\circ}$ ) Comunicações Auxiliares. Aliás, foi fácil nomear esses fatores, haja vista a coerência da natureza das variáveis que integram cada um deles.

\section{Resultados da segunda questão}

Acerca da segunda questão, na Tabela 3 , encontramse os resultados da análise do alfa de Cronbach aplicada sobre as escalas de cada um dos seis fatores detectados. À parte, foi examinada a variação do alfa provocada pela exclusão de cada variável (Norusis, 1993). Mas apenas a escala do fator 5 (Profissionalismo e Responsabilidade Profissional) tinha na sua variável V19 a possibilidade de reduzido incremento no alfa com a sua exclusão. Assim, optou-se por manter essa escala em sua composição original.

O critério aqui adotado, dentre vários existentes, é que um alfa igual ou maior a 0,70 espelha suficiente fidedignidade de uma escala, consoante Nunnaly (1978). Temse em mente, todavia, a discordância de Pedhazur e Schmelkin (1991) com o estabelecimento de padrões específicos de fidedignidade, alegando que eles freqüentemente tendem a assumir vida própria e acabam aplicados sem preocupação com os fundamentos que os geraram. Os dados da Tabela 3 evidenciam que a fidedignidade de cinco das seis escalas dos fatores da QPC é aceitável. Mesmo a escala do sexto fator, com alfa igual a 0,69, está quase dentro do patamar mínimo de Nunnaly (1978). Desse modo, para a segunda questão, têm-se como praticamente fidedignas as escalas da QPC para os seis fatores prevalecentes nesta pesquisa. Já no estudo dos fatores de experiência de Brown e Swartz (1989), na operacionalização de sua QPC, os alfas variavam de 0,64 a

Tabela 1 - Análise fatorial da QPC extraindo-se 6 fatores dos 43 atributos

\begin{tabular}{|c|c|c|c|c|c|c|}
\hline \multirow{2}{*}{ Fator } & \multicolumn{3}{|c|}{ Situação após a extração dos fatores } & \multicolumn{3}{c|}{ Situação após a rotação dos fatores } \\
\cline { 2 - 7 } & Autovalor & \% da variância & \% cumulativa & Autovalor & \% da variância & $\%$ cumulativa \\
\hline 1 & 18,125 & 42,152 & 42,152 & 9,823 & 22,845 & 22,845 \\
2 & 2,688 & 6,251 & 48,403 & 5,088 & 11,832 & 34,676 \\
3 & 1,710 & 3,977 & 52,380 & 3,776 & 8,782 & 43,458 \\
4 & 1,281 & 2,978 & 55,358 & 2,917 & 6,783 & 50,241 \\
5 & 1,081 & 2,513 & 57,872 & 2,598 & 6,041 & 56,282 \\
6 & 1,019 & 2,369 & 60,241 & 1,702 & 3,959 & 60,241 \\
\hline
\end{tabular}


Tabela 2 - Matriz incompleta de fatores rotacionados extraindo-se 6 fatores dos 43 atributos da QPC (apenas os coeficientes acima de 0,4 )

\begin{tabular}{|c|c|c|c|c|c|c|c|c|}
\hline \multirow{2}{*}{ Variável - Mnemônico } & \multirow{2}{*}{ Média } & \multirow{2}{*}{$\begin{array}{l}\text { Desvio- } \\
\text { padrão }\end{array}$} & \multicolumn{6}{|c|}{ Fator } \\
\hline & & & $1^{\circ}$ & $2^{\circ}$ & $3^{\circ}$ & $4^{\circ}$ & $5^{\circ}$ & $6^{\circ}$ \\
\hline V01 - médico ouvir o paciente. & 4,60 & 0,75 & 0,76 & & & & & \\
\hline V02 - médico dar informações sobre a saúde. & 4,33 & 0,97 & 0,76 & & & & & \\
\hline V03 - médico ser cuidadoso para explicar o que fazer. & 4,41 & 0,92 & 0,72 & & & & & \\
\hline V04 - médico ser minucioso no atendimento. & 4,23 & 1,01 & 0,81 & & & & & \\
\hline V05 - médico dedicar tempo ao atendimento. & 4,44 & 0,91 & 0,71 & & & & & \\
\hline V06 - médico examinar bem antes de concluir. & 4,37 & 0,97 & 0,76 & & & & & \\
\hline V07 - médico inspirar total confiança. & 4,40 & 0,97 & 0,72 & & & & & \\
\hline V08 - médico ter real interesse pela pessoa. & 4,24 & 0,92 & 0,71 & & & & & \\
\hline V09 - médico dar total atenção. & 4,53 & 0,85 & 0,73 & & & & & \\
\hline V10 - médico tratar com respeito. & 4,78 & 0,59 & 0,56 & & & & & \\
\hline V11 - médico explicar as razões dos exames. & 4,43 & 0,98 & 0,63 & & & & & \\
\hline V12 - recepcionistas terem interesse pessoal. & 3,18 & 1,17 & & & 0,72 & & & \\
\hline V13 - recepcionistas conhecerem individualmente. & 2,81 & 1,34 & & & 0,64 & & & \\
\hline V14 - recepcionistas serem corteses. & 4,10 & 1,04 & & & 0,68 & & & \\
\hline V15 - recepcionistas do médico serem flexíveis. & 3,74 & 1,13 & & & 0,77 & & & \\
\hline V16 - recepcionistas agirem de forma profissional. & 4,10 & 1,03 & & & 0,63 & & & \\
\hline V17 - recepcionistas interessadas em atender. & 2,90 & 1,22 & & & 0,66 & & & \\
\hline V18 - médico receitar só remédios necessários. & 4,62 & 0,77 & 0,48 & 0,51 & & & & \\
\hline V19 - médico não pedir exames desnecessários. & 4,62 & 0,84 & 0,43 & 0,45 & & & 0,48 & \\
\hline V20 - médico não levar a riscos desnecessários. & 4,56 & 0,88 & 0,44 & 0,57 & & & 0,45 & \\
\hline V21 - médico não ter como prioridade o dinheiro. & 4,38 & 1,07 & & & & & 0,64 & \\
\hline V22 - médico não priorizar conversar com funcionários. & 4,43 & 1,02 & & & & & 0,65 & \\
\hline V23 - médico admitir quando não sabe. & 4,31 & 1,01 & & & & & & \\
\hline V24 - nada haver a melhorar no tratamento. & 4,16 & 1,01 & 0,43 & 0,46 & & & & \\
\hline V25 - médico explicar os problemas de saúde. & 4,49 & 0,90 & 0,59 & 0,42 & & & & \\
\hline V26 - médico ter elevada qualificação. & 4,57 & 0,78 & & 0,73 & & & & \\
\hline V27 - médico não cometer erros. & 4,50 & 0,93 & 0,51 & 0,57 & & & & \\
\hline V28 - médico se manter atualizado na medicina. & 4,55 & 0,84 & & 0,69 & & & & \\
\hline V29 - médico dar alternativas de tratamento. & 4,51 & 0,91 & 0,54 & 0,59 & & & & \\
\hline V30 - médico raramente fazer esperar. & 3,90 & 1,20 & & & & 0,52 & & \\
\hline V31 - horários do consultório serem cômodos. & 4,23 & 0,96 & & & & 0,60 & & \\
\hline V32 - consultório ser de fácil acesso. & 4,19 & 1,12 & & & & 0,62 & & \\
\hline V33 - médico atuar em hospital de fácil acesso. & 3,82 & 1,27 & & & & 0,59 & & 0,49 \\
\hline V34 - ser fácil e rápido marcar consulta. & 3,98 & 1,15 & & & & 0,54 & & \\
\hline V35 - médico ser honesto ao cobrar. & 4,77 & 0,60 & & & & & & \\
\hline V36 - médico falar com clareza. & 4,67 & 0,76 & 0,51 & & & & 0,41 & \\
\hline V37 - médico ter interesse sincero. & 4,38 & 0,92 & 0,63 & & & & & \\
\hline V38 - médico explicar pessoalmente os exames. & 4,64 & 0,77 & 0,57 & & & & & \\
\hline V39 - sala de espera com informações de saúde. & 3,49 & 1,35 & & & & & & 0,64 \\
\hline V40 - médico fornecer folhetos explicativos. & 3,09 & 1,42 & & & & & & 0,48 \\
\hline V41 - médico tomar as decisões que Ihe cabem. & 4,25 & 1,02 & 0,46 & 0,46 & & & & \\
\hline V42 - médico atualizado com a tecnologia médica. & 4,39 & 0,90 & & 0,72 & & & & \\
\hline V43 - médico disponível em uma emergência. & 3,88 & 1,31 & & & & & & \\
\hline
\end{tabular}


0,93 . Por conseguinte, os alfas extremos desta pesquisa são ligeiramente superiores àqueles originais, reforçando a procedência da estrutura ora reconhecida para as escalas.

\section{Resultados da terceira questão}

A terceira questão foi avaliada sob duas formas de validade: convergente e discriminante (Pedhazur e Schmelkin, 1991, p. 73-75). Como variável dependente da análise de regressão linear: a) na convergente, teve-se a Avaliação Global da QPC; b) na discriminante, teve-se a Satisfação Global com Serviços de Consultório. Em ambos os casos, como variáveis independentes entraram índices para cada um dos seis fatores da QPC prevalecentes nesta pesquisa (média dos escores de suas variáveis respectivas). $\mathrm{Na}$ checagem das premissas (normalidade, hemocedasticidade e linearidade) para a análise de regressão (Hair Jr. et al., 1998), somente as variáveis dos índices dos fatores $3 \mathrm{e}$ 4 passaram no teste de normalidade univariada de Kolmogorov-Smirnov (com a correção de significância de Lilliefors). Todavia, quando a amostra é grande, como aqui, quase todo teste de ajustamento rejeitará a hipótese nula de normalidade. Não obstante, para a maioria dos testes estatísticos, é suficiente ter-se a distribuição como aproximadamente normal (Norusis, 1993). Isso permitiu aplicar a análise de regressão linear múltipla.

Os resultados para a validade convergente estão na Tabela 4. Há um significativo e elevado coeficiente de determinação ajustado: 79\%, ou seja, os seis índices dos fatores da QPC explicam alta proporção da variância da Avaliação
Global da QPC. Apenas o índice do fator 6 não tem influência significativa na relação linear existente. Essas estimativas não foram prejudicadas por alta multicolinearidade entre as variáveis independentes, pois o menor valor de tolerância dentre as seis variáveis independentes foi 0,3 , bem acima do limiar de 0,1 (Hair Jr. et al., 1998). Além disso, os coeficientes de regressão padronizados da Tabela 4 proporcionam uma ordenação decrescente - com cautela - da força dos índices dos fatores significativos da QPC como preditores a Avaliação Global da QPC: $1^{\circ}$ ) 1 Interações do Médico e Diagnóstico, com coeficiente de 0,$\left.35 ; 2^{\circ}\right) 3$ - Interações de Auxiliares, com coeficiente de 0,$\left.30 ; 3^{\circ}\right) 2$ - Competência Profissional, com coeficiente de 0,$\left.20 ; 4^{\circ}\right) 5$ - Profissionalismo e Responsabilidade Profissional, com coeficiente de 0,14 ; e $\left.5^{\circ}\right) 4$ - Comodidade de Horários e de Localização, com coeficiente de 0,11.

$\mathrm{Na}$ validade discriminante, os resultados da análise de regressão foram essencialmente semelhantes aos da análise correspondente para a validade convergente, motivo pelo qual deixam de ser apresentados neste restrito espaço. Isso indica que os construtos da QP e satisfação, sob o ponto de vista da mensuração, na verdade, são indistintos na esfera estudada de serviços de consultórios médicos. Assim, a resposta à terceira questão é parcialmente afirmativa. Há validade convergente nas escalas da QPC dos fatores 1 a 5, mas carece de validade a escala do fator 6. Porém não há validade discriminante das escalas da QPC desses fatores todos em relação à Satisfação Global com Serviços de Consultório.

Tabela 3 - Análise de fidedignidade das escalas dos seis fatores da QPC detectados

\begin{tabular}{|l|c|}
\hline \multicolumn{1}{|c|}{ Fator da escala } & Alfa padronizado do fator \\
\hline $1^{\circ}$ - Interações do Médico e Diagnóstico & 0,957 \\
$2^{\circ}$ - Competência Profissional & 0,939 \\
$3^{\circ}$ - Interações de Auxiliares & 0,879 \\
$4^{\circ}$ - Comodidade de Horários e Localização & 0,792 \\
$5^{\circ}$ - Profissionalismo e Responsabilidade Profissional & 0,763 \\
$6^{\circ}$ - Comunicações Auxiliares & 0,688 \\
\hline
\end{tabular}

Tabela 4 - Análise de regressão da Avaliação Global da QPC sobre os seis índices dos fatores da QPC

\begin{tabular}{|l|l|c|c|c|}
\hline \multicolumn{1}{|c|}{$\begin{array}{c}\text { Variáveis } \\
\text { independentes }\end{array}$} & $\begin{array}{c}\text { Coeficiente de } \\
\text { regressão }\end{array}$ & $\begin{array}{c}\text { Coeficiente de } \\
\text { regressão padronizado }\end{array}$ & $\begin{array}{c}\text { Estatística } \\
\text { t }\end{array}$ & $\begin{array}{c}\text { Significância } \\
\text { de t }\end{array}$ \\
\hline Constante & $-0,799$ & & $-3,815$ & 0,000 \\
Índice do Fator 1 & 0,425 & 0,354 & 5,199 & 0,000 \\
Índice do Fator 2 & 0,218 & 0,204 & 2,776 & 0,006 \\
Índice do Fator 3 & 0,256 & 0,304 & 6,960 & 0,000 \\
Índice do Fator 4 & $7,900 \mathrm{E}-02$ & 0,111 & 2,361 & 0,020 \\
Índice do Fator 5 & 0,131 & 0,136 & 2,751 & 0,007 \\
Índice do Fator 6 & $9,490 \mathrm{E}-03$ & 0,015 & 0,361 & 0,719 \\
\hline
\end{tabular}




\section{CONCLUSÕES E IMPLICAÇÕES}

Os resultados alcançados neste estudo, até o momento originais no Brasil, podem ajudar na compreensão científica da Qualidade Percebida de Consultório (QPC) e em aplicações na prática médica de consultório. Despontaram seis fatores (Tabela 3), sendo cinco deles válidos subjacentes à QPC, englobando 40 atributos significativos (Tabela $1 \mathrm{e}$ Quadro 2). Embora não tenha prevalecido a solução original norte-americana com nove fatores, o interessante é que, por trás dos seis fatores ora detectados, estão agrupamentos de variáveis em fatores idênticos, ou praticamente idênticos, em relação àqueles propostos originalmente por Brown e Swartz (1989). Isso sugere que o domínio total, anteriormente por eles proposto, tem uma configuração estrutural semelhante nas amostras norte-americana e brasileira - algo fundamental à vista da pretensão científica de descobrir relações mais e mais gerais.

Quanto à fidedignidade, ela é praticamente apropriada em todas as seis escalas dos fatores prevalecentes neste estudo para a QPC (Tabela 3). A validade convergente é expressiva, em que os seis índices dos fatores explicam alta proporção (79\%) da variância da Avaliação Global da QPC. Somente o índice do fator 6 (Comunicações Auxiliares) não tem influência significativa na relação linear existente (Tabela 4). Mas não há validade discriminante entre a escala da QPC e satisfação global, contrariando Bolton e Drew (1991a), La'Barbera e Mazursky (1983) e Oliver (1997), o que traz importante e positiva implicação profissional. Pacientes que percebem alta qualidade nos serviços de um médico no consultório devem estar também muito satisfeitos, de onde as consequiências auspiciosas de um e outro construto estarem ao mesmo tempo viabilizadas.

Não se discute que os pacientes são o objetivo último e a razão de ser de qualquer sistema de saúde. O difícil consiste em que os pacientes consideram um conjunto bem amplo e variado de fatores e atributos como QPC (Quadro 2). Por esse motivo, uma medida da QPC fornecida por essa escala indica o grau do sucesso do médico em atender às necessidades e desejos de seus pacientes no consultório. A escala da QPC contém fatores significativos e atributos tanto de qualidade técnica quanto funcional (Grönroos, 1990) esta última, equiparada ao domínio interpessoal e de amenidades esquematizado por Donabedian (1980, 1985).

Cabe um alerta aos profissionais de saúde porque, ao menos em parte, tendem a ser distintas as considerações nas perspectivas de paciente e médico (Carson et al., 1998). As avaliações humanas de qualidade são subjetivas, influenciadas pelo particular referencial do avaliador - como valores, conhecimentos e interesses. Em conseqüência, a chance de os profissionais de saúde colocarem-se no lugar dos pacientes e substituírem com acerto as avaliações destes é normalmente pequena. O jeito é dar voz própria ao paciente. Óbvio é que limites precisam ser impostos à utilização da perspectiva dos pacien- tes no direcionamento do trabalho nos serviços de saúde. O paciente quase sempre conhece bem pouco os detalhes da qualidade técnica (Donabedian, 1985). O paciente típico não poderia, por exemplo, apontar falhas em um esquema de medicação. Mas, na qualidade funcional de serviços de saúde, o paciente pode, certamente, avaliar o que lhe é oferecido.

Com efeito, embora muitas fontes devam ser usadas para delinear o esforço de melhoria de qualidade de serviços de saúde, Berwick, Godfrey e Roessner (1990) assinalam que a melhor das fontes está nos próprios pacientes. Uma posição que não surpreende à vista da teoria geral de gestão da qualidade, como filosofia gerencial orientada para

\section{A CHANCE DE OS PROFISSIONAIS DE}

\section{SAÚDE COLOCAREM-SE NO LUGAR}

\section{DOS PACIENTES E SUBSTITUÍREM}

\section{COM ACERTO AS AVALIAÇÕES \\ DESTES É NORMALMENTE PEQUENA.}

o cliente e que toma este como ponto de partida para o alinhamento de toda a gestão (Jun, Peterson e Zsidisin, 1998). Essa visão merece maior acolhida no meio de saúde, à mercê da compreensão de seu real significado e impacto. Basta verificar que, nos Estados Unidos, naquilo que pode ser o maior estudo sobre saúde fora do censo, está sendo implementado um sistema nacional privado de pesquisa com a avaliação dos pacientes sobre o desempenho não-clínico (vale dizer, qualidade funcional) dos médicos. Espera-se que os planos de saúde comprem essas informações e as ofereçam a seus clientes (Duke, 2000).

Diante de um cenário desses, a ligação entre médico e paciente reclama uma mudança de foco, de uma base transacional - dita como uma pseudo-interação - para um genuíno relacionamento, marcado por maior envolvimento do paciente e compartilhamento bidirecional de informações (Johnson e Ramaprasad, 2000). Em particular, apesar de suas limitações, a escala da QPC aqui delineada enseja aos médicos e organizações prestadoras de serviços um meio para aprimoramento mais criterioso do trabalho de consultório. Logo, ainda que caiba aperfeiçoar tal escala, sobretudo impõe-se aplicá-la para que produza benefícios (Anderson, 1995). Indo além, dado os enormes ganhos potenciais que de esforços de pesquisa podem advir, são necessárias mais iniciativas para jogar luz sobre a QP em saúde. Afinal, serviços de alta qualidade, tal qual bens, ocupam posição competitiva privilegiada no mercado - o que não é diferente com serviços de saúde (Quality, 2001). Espera-se que este artigo seja uma contribuição a realçar a premência dessa orientação. 
LIMITAÇÕES DESTE ESTUDO

E DIREÇÕES PARA O FUTURO

Neste estudo, ocorreu reduzida variação nos escores dos atributos da QPC, o que pode ter prejudicado a robustez das análises. Logo, em outras oportunidades de pesquisa, deve-se buscar trabalhar com amostras mais heterogêneas, embora sejam conhecidas as restrições de recursos no Brasil para custear pesquisas de maior envergadura e a dificuldade em obter cooperação de organizações e profissionais de saúde.

Incidiu sobre a pesquisa um potencial erro de nãoresposta, pois a maior parte dos pacientes (67\% do total de 1.050 contatados) selecionados para participar da

Quadro 2 - Composição final da escala da QPC: fatores e variáveis

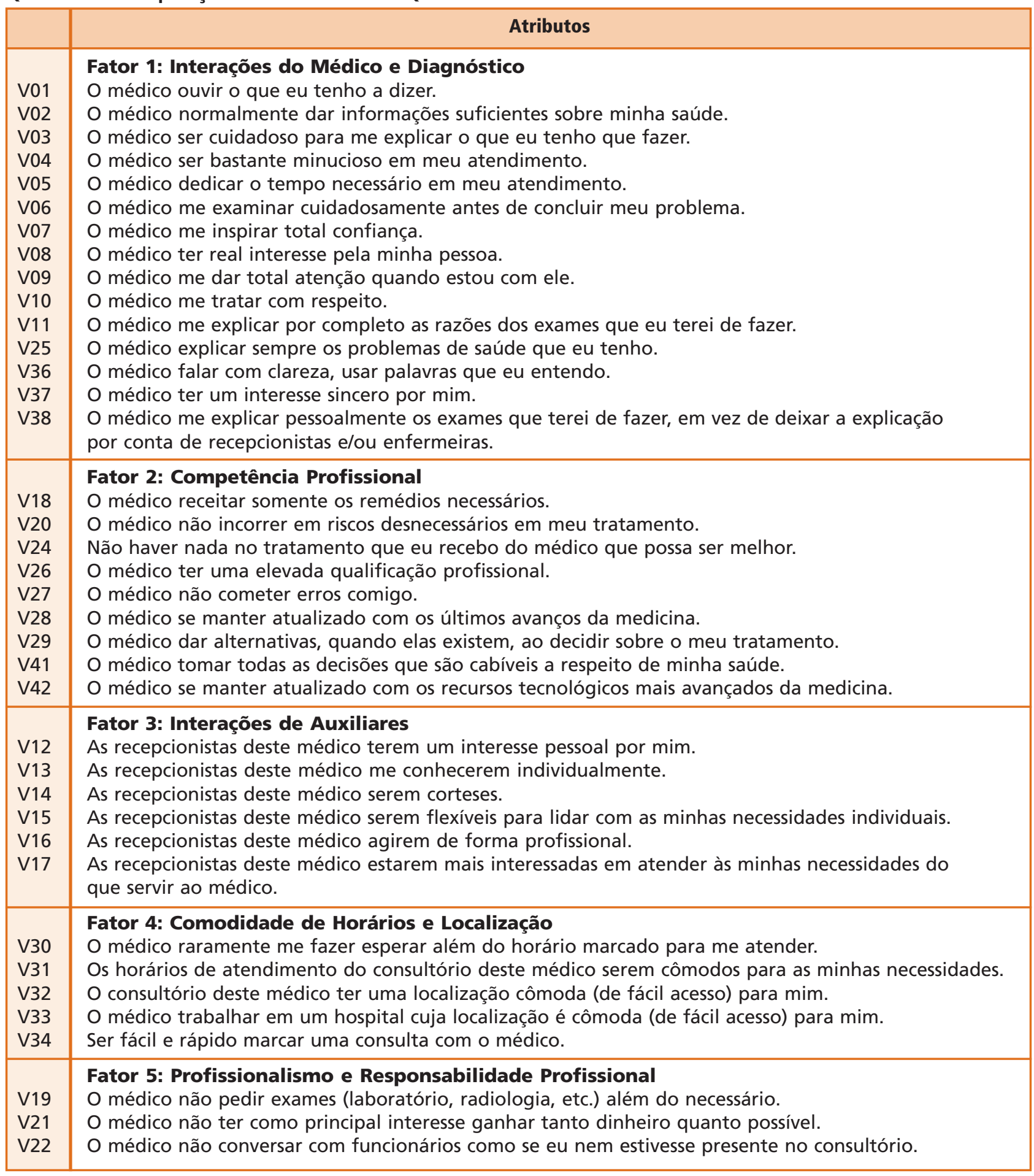


amostra não respondeu ao questionário. Resta a dúvida se aqueles que responderam diferem substantivamente daqueles que não responderam em termos de variáveis relevantes para o estudo (Hayes, 1992). Em outras pesquisas, devem ser implementadas ações para elevar a taxa de respostas e estimar-se estatisticamente a incidência desse viés.

Generalização dos resultados foi outra limitação da pesquisa (Kerlinger, 1973), já que os pacientes estudados foram todos provenientes de um mesmo plano de saúde e praticamente de uma cidade apenas - Belo Horizonte. Recomendável seria promover o teste das questões desta pesquisa com pacientes (como os particulares) e médicos de outras categorias, de outras modalidades organizacionais (como as seguradoras), com outros fins (visando lucro) e de outras cidades e regiões brasileiras. $\bigcirc$

NOTA

Pesquisa realizada com o apoio financeiro da Fundação de Amparo à Pesquisa do Estado de Minas Gerais.

\section{REFERÊNCIAS BIBLIOGRÁFICAS}

ANDERSON, Elizabeth A. Measuring service quality at a university health clinic. International Journal of Health Care Quality Assurance, v. 8, n. 2, p. 32-37, 1995

BERWICK, Donald M., GODFREY, A. Blanton, ROESSNER, Jane. Curing health care - new strategies for quality improvement: a report on the National Demonstration Project on Quality Improvement in Health Care. San Francisco : Jossey-Bass, 1990.

BLENDON, Robert J. et al. Physician's views on quality of care: a five-country comparison. Health Affairs, v. 20, n. 6, p. 29, Mar. 2001.

BOLTON, Ruth N., DREW, James H. A longitudinal analysis of the impact of service changes on customer attitudes. Journal of Marketing, v. 55, n. 1, p. 1-9, Jan. 1991a.

BOLTON, Ruth N., DREW, James H. A multistage model of customer's assessments of service quality and value. Journal of Consumer Research, v. 17, n. 1, p. 375-384, Mar. 1991b.

BROWN, Stephen W., SWARTZ, Teresa A. A gap analysis of professional service quality. Journal of Marketing, v. 53, n. 2, p. 92-98, Apr. 1989.

CARSON, Paula P., CARSON, Kerry D., ROE, C. William. Toward understanding the patient's perception of quality. Health Care Supervisor, v. 16, n. 3, p. 36-42, 1998.

CHURCHILL, Gilbert A. Marketing research: methodological foundations. Fort Worth : Dryden Press, 1991.

CLARK, Liana R. How do we get back our humanity? Medical Economics, v. 78, n. 9, p. 96-98, May 2001.

CRONIN, J. Joseph, TAYLOR, Steven A. Measuring service quality: a reexamination and extension. Journal of Marketing, v. 56, n. 3, p. 55-68, July 1992.

DONABEDIAN, Avedis. Explorations in quality assessment and monitoring. Ann Arbor : Health Administration Press, 1980. V. I : The definition of quality and approaches to its assessment.

DONABEDIAN, Avedis. Explorations in quality assessment and monitoring. Ann Arbor : Health Administration Press, 1985. V. II: The methods and findings of quality assessment and monitoring: an illustrated analysis.

DUKE, Suzanne. Patients nationwide will rate your nonclinical skills. Medical Economics, v. 77, n. 16, p. 22, Aug. 2000.

FORD, Robert C., BACH, Susan A., FOTTLER, Myron D. Methods of measuring patient satisfaction in health care organizations. Health Care Management Review, v. 22, n. 2, p. 74-89, 1997.
GARVIN, David A. What does "product quality" really means. Sloan Management Review, v. 26, n. 1, p. 23-43, Fall 1984.

GARVIN, David A. Afterword: reflections on the future. In: BERWICK, Donald M., GODFREY, A. Blanton, ROESSNER, Jane. Curing health care - new strategies for quality improvement: a report on the National Demonstration Project on Quality Improvement in Health Care. San Francisco : Jossey-Bass, 1990. p. 159-165.

GRÖNROOS, Christian. Service management and marketing managing the moments of truth in service competition. Lexington : Lexington Books, 1990.

HAIR JR., Joseph F. et al. Multivariate data analysis with readings. 5. ed. New York: Macmillan, 1998.

HAYES, Bob E. Measuring customer satisfaction development and use of questionnaires. Milwaukee : ASQC Quality Press, 1992.

HOWARD, John A. Consumer behavior: application of theory. New York: McGraw-Hill, 1977.

JOHNSON, Grace L., RAMAPRASAD, Arkalgud. Patientphysician relationships in the information age. Marketing Health Services, v. 20, n. 1, p. 20-27, Spring 2000.

JOHNSTON, David M., LUCE, Fernando B. As escalas Servqual e Servperf no setor de serviços bancários. In: ENCONTRO ANUAL DA ASSOCIAÇÃO NACIONAL DOS PROGRAMAS DE PÓS-GRADUAÇÃO EM ADMINISTRAÇÃO 20, 1996, Angra dos Reis. Anais... Rio de Janeiro : Anpad, 1996. Marketing, p. 91-108.

JUN, Minjoon, PETERSON, Robin T., ZSIDISIN, George A. The identification and measurement of quality dimensions in health care: focus groups interview results. Health Care Management Review, v. 23, n. 4, p. 81-96, 1998.

KERLINGER, Frederick N. Foundations of behavioral research. New York : Holt, Rinehart and Winston, 1973.

KINNEAR, Thomas C., TAYLOR, James R. Marketing research: an applied approach. New York : McGraw-Hill, 1991.

LA'BARBERA, Priscilla A., MAZURSKY, David. A longitudinal assessment of consumer satisfaction/dissatisfaction: the dynamic aspect of the cognitive process. Journal of Marketing Research, v. 20, n. 4, p. 393-404, 1983.

MALHOTRA, Naresh K. Marketing research: an applied orientation. Upper Saddle River : Prentice Hall, 1996.

MONROE, Kent B., KRISHNAN, R. The effect of price on subjective product evaluations. In: JACOBY, Jacob, OLSON, Jerry C. Perceived quality: how consumers view stores and merchandise. Lexington : Lexington Books, 1985. p. 209-232.

NORUSIS, Marisa J. SPSS for Windows: base system user's guide. Release 6.0. Chicago : SPSS, 1993.
NUNNALY, Jum C. Psychometric theory. 2. ed. New York: McGraw-Hill, 1978.

O'CONNOR, Stephen J., TRINH, Hanh Q., SHEWCHUK, Richard M. Perceptual gaps in understanding patient expectations for health care service quality. Health Care Management Review, v. 25, n. 2, p. 7-23, Spring 2000.

OLIVER, Richard. Satisfaction: a behavioral perspective on the consumer. Boston : Irwin McGraw-Hill, 1997.

PARASURAMAN, A., ZEITHAML, Valarie A., BERRY Leonard L. A conceptual model of service quality and its implications for future research. Journal of Marketing, v. 49, n. 4, p. 41-50, Fall 1985.

PARASURAMAN, A., ZEITHAML, Valarie A., BERRY, Leonard L. SERVQUAL: a multiple-item scale for measuring consumer perceptions of service quality. Journal of Retailing, v. 64, n. 1, p. 12-39, Spring 1988.

PEDHAZUR, Elazar J., SCHMELKIN, Liora P. Measurement, design and analysis: an integrated approach. Hillsdale : Lawrence Erlbaum, 1991.

PERRIEN, Jean, CHÉRON, Emmanuel, ZINS, Michel. Recherche en marketing: méthodes et décisions. Québec : Gaetan Morin, 1986

PRETZER, Michael. What patients don't know about the quality of their health care. Medical Economics, v. 78, n. 10, p. 26-28, May 2001.

QUALITY and service are paramount: a survey of healthcare purchasing trends. Healthcare Financial Management, v. 55, n. 6, p. 72-76, June 2001.

SCHEWCHUK, Richard M., O'CONNOR, Stephen J. WHITE, Joseph B. In search of service quality measures: some questions regarding psychometric properties. Health Services Management Research, v. 4, n. 1, p. 65-75, 1991.

TAYLOR, Steven A. Distinguishing service quality from patient satisfaction in developing health care marketing strategies. Hospital \& Health Services Administration, v. 39, n. 2, p. 221-236, Summer 1994.

TAYLOR, Steven A., CRONIN, J. Joseph. Modeling patient satisfaction and service quality. Journal of Health Care Marketing, v. 14, n. 1, p. 34-44, 1994.

TULL, Donald S., HAWKINS, Del I. Marketing research: measurement \& method. New York : Macmillan, 1990.

URDAN, André T., MAGRO, Alexandre S. Uma comparação empírica de duas bases de mensuração da qualidade de serviços percebida: desconformidade versus desempenho. In: ENCONTRO ANUAL DA ASSOCIAÇÃO NACIONAL DOS PROGRAMAS DE PÓS-GRADUAÇ̃̃O EM ADMINISTRAÇÃO, 20, 1996, Angra dos Reis. Anais... Rio de Janeiro : Anpad, 1996. p. 47-69. 